\title{
Anal gland adenocarcinoma in situ with pagetoid spread: a case report
}

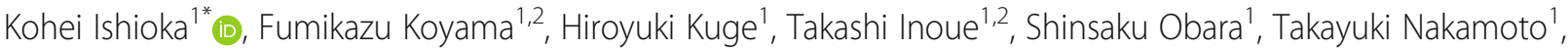 \\ Yoshiyuki Sasaki', Yasuyuki Nakamura', Maiko Takeda ${ }^{3}$, Chiho Ohbayashi ${ }^{3}$, Masamitsu Kuwahara ${ }^{4}$ \\ and Masayuki Sho ${ }^{1}$
}

\begin{abstract}
Background: Anal gland carcinoma with perianal Paget's disease is rare, and anal gland carcinoma in situ is extremely rare. No cases of anal gland carcinoma in situ with pagetoid spread have been previously reported.

Case presentation: Physical examination in a 75-year-old woman revealed an erythematous, inflamed, perianal skin lesion. Neither colposcopy, cystoscopy, colonoscopy, computed tomography, nor magnetic resonance imaging showed evidence of malignant genitourinary or gastrointestinal lesions. Histopathological examination of a biopsy specimen showed many Paget's cells in the perianal skin lesion and no malignant cells in the rectal or vaginal mucosa. Therefore, primary extramammary Paget's disease of the anogenital region was suspected, and we performed anus-preserving wide local excision. However, immunohistochemistry revealed a diagnosis of secondary extramammary Paget's disease due to adenocarcinoma arising from the anal gland. We therefore proceeded with a radical operation. Histopathological examination showed no residual cancer cells. The final diagnosis was anal gland adenocarcinoma in situ with pagetoid spread in the perianal skin.

Conclusions: This is the first case report of anal gland adenocarcinoma in situ with pagetoid spread. We recommend immunohistochemical analysis of biopsy and locally resected specimens to obtain an accurate diagnosis and determine the appropriate treatment when there is no visible tumor.
\end{abstract}

Keywords: Anal gland adenocarcinoma, Adenocarcinoma in situ, Perianal Paget's disease

\section{Background}

Perianal Paget's disease (PPD) is rare. There are two types of PPD: primary and secondary. When an underlying adenocarcinoma is present, PPD usually represents intraepidermal extension of an invasive carcinoma from an adjacent internal organ and is generally regarded as secondary [1].

There are some reports of pagetoid spread of adenocarcinoma in situ of the cervix [2] and pagetoid changes in urothelial carcinoma in situ [3, 4]. However, there are no reports of anal gland adenocarcinoma in situ with pagetoid spread.

\footnotetext{
* Correspondence: kohei.@@naramed-u.ac.jp

'Department of Surgery, Nara Medical University, 840 Shijo-cho, Kashihara, Nara 634-8522, Japan

Full list of author information is available at the end of the article
}

Herein, we report the first documented case of anal gland carcinoma in situ with pagetoid spread.

\section{Case presentation}

A 75-year-old woman was admitted to our hospital complaining of itching around her anus. She had a history of sigmoidectomy for diverticulitis 6 years prior and a past history of Sjögren's syndrome. She had noted a reddish skin lesion around her anus over the past several years. She reported no change in bowel habits, no gastrointestinal symptoms, no weight loss, and no family history of malignancy.

Physical examination revealed an erythematous, inflamed skin lesion in the perianal region (Fig. 1), but a normal vagina and rectum. Neither colposcopy, cystoscopy, nor colonoscopy showed evidence of a visible lesion or any abnormality of the cervix, bladder, or rectum (Fig. 2a, b). Computed tomography 


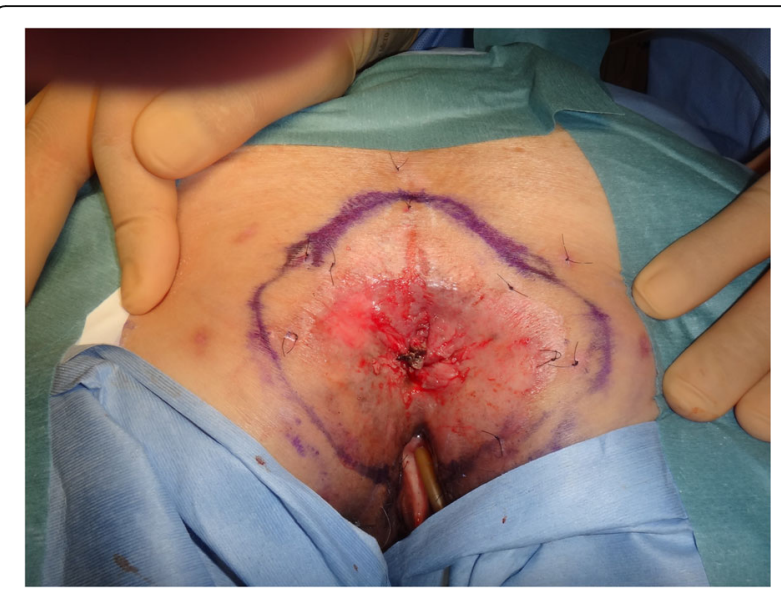

Fig. 1 Macroscopic view of the perianal skin. A $60 \times 75-\mathrm{mm}$ pagetoid spreading lesion is visible around the anus

and magnetic resonance imaging showed no evidence of malignancy in the genitourinary or gastrointestinal tracts. Histopathological examination of biopsy specimens showed many Paget's cells within intraepithelial lesions of the perianal skin but no malignant cells in the rectal or vaginal mucosa. Therefore, primary extramammary Paget's disease (EMPD) of the anogenital region was suspected. We performed anal-preserving wide local excision deep to the subcutaneous fat with $1-\mathrm{cm}$ negative margin from the positive sites confirmed by frozen section examination and mucosal resection of the anal canal that was extended $1 \mathrm{~cm}$ proximal to the dentate line of the anal canal. Reconstruction was performed using a bilobed gluteal fold flap (Fig. 3a, b).

Histopathological examination of the resected specimen showed Paget's cells, characterized by clear cytoplasm and large pleomorphic nuclei, within the epidermis. Immunohistochemical analysis revealed that the Paget's cells were positive for cytokeratin
(CK) 7, CK20, and caudal-related homeobox gene nuclear transcription factor (CDX) 2 and negative for gross cystic disease fluid protein (GCDFP) 15 (Fig. 4a-e). These data suggested that her perianal skin lesion was secondary EMPD arising from the rectum, although there was no lesion found in the rectum. Additional histopathological examination of the resected specimen revealed well-differentiated adenocarcinoma in an anal gland, continuous with the Paget's cells in the anoderm, but limited in the basement membrane without a desmoplastic change (Figs. 5a, b and 6a-e). Immunohistochemical staining of the resected specimen revealed secondary EMPD due to adenocarcinoma arising from the anal gland.

Because of the possibility of residual adenocarcinoma in the anal gland, possibly extending to the sphincter, we performed a radical laparoscopy-assisted abdominoperineal resection. Any reconstructive plastic surgery was not needed. Histopathological examination revealed no residual cancer cells in the resected specimen and no lymph node metastasis. The final diagnosis was anal gland adenocarcinoma in situ with pagetoid spread in the perianal skin.

\section{Discussion}

PPD was defined as intraepithelial adenocarcinoma of the perianal skin and is classified into two subgroups: primary and secondary [5]. Primary PPD originates from the epidermis or skin appendages, including the eccrine glands, apocrine glands, ectopic mammary-like glands, and epidermal pluripotent stem cells. Secondary PPD, also known as pagetoid phenomenon, is a metastatic tumor derived from underlying carcinoma cells [1]. Immunohistochemical staining for GCDFP15, CDX2, CK7, and CK20 are especially useful in differentiating the type and the origins of EMPD. GCDFP15 is considered as apocrine epithelium-specific tissue marker [1], and

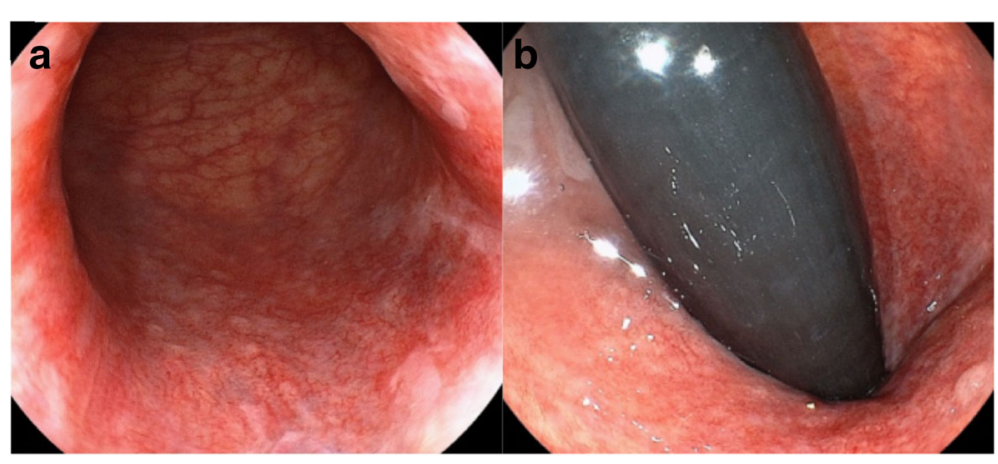

Fig. 2 Colonoscopy. There is no visible lesion in the rectum or anal canal. a Ordinary observation. b Reversal observation 


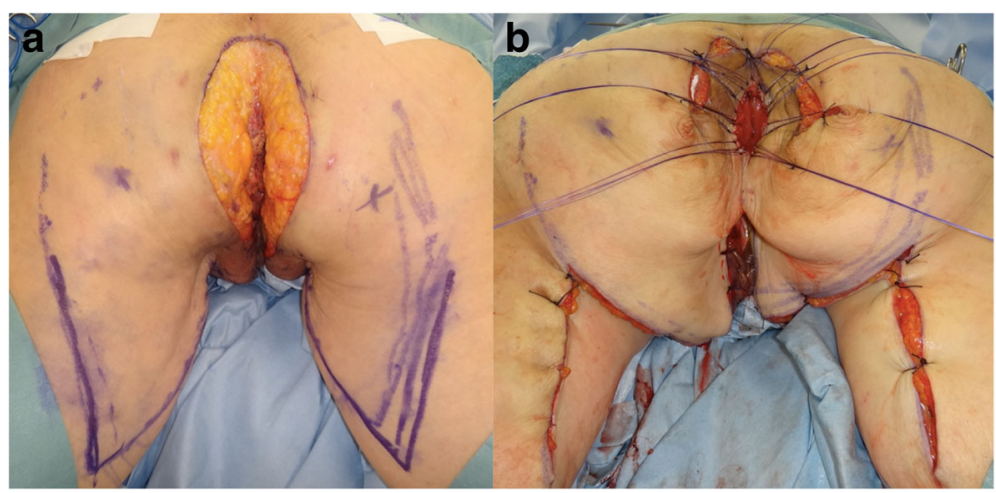

Fig. 3 Surgical findings. a Anal-preserving wide local excision deep to the subcutaneous fat. b Reconstruction using a bilobed gluteal fold flap

negative in secondary EMPD [6]. The expression of CDX2 is a sensitive and specific marker for the secondary type, arising from anorectal or colonic adenocarcinoma [7], but CDX2 fails to distinguish primary disease from EMPD secondary to urothelial or prostatic malignancy [8]. Many anal gland adenocarcinomas stained for CK7 but did not stain for CK20, similar to the normal anal gland [9-11]. However, several cases of secondary type arising from anal gland carcinoma showed CK7 positive and CK20 positive [12-14].

Approximately $50 \%$ of patients with anal-margin Paget's disease harbor a colorectal neoplasm mandating full colonoscopy for complete evaluation [15]. In our patient, there was no visible gastrointestinal, genital, or urinary tumor, and we initially suspected primary PPD. However, immunohistochemical analysis (CK7 +, CK20 +, GCDFP15 -, CDX2 +) suggested secondary EMPD. This discrepancy led us to perform additional histopathological studies on the resected specimen, which revealed the original site of the Paget's disease as an in situ, well-differentiated adenocarcinoma in an anal gland.

Anal crypt glands, which lie at the level of the dentate line, can extend into the longitudinal layer after penetrating the internal sphincter $[16,17]$. Thus, combined resection of the anal sphincter at the site of the malignancy is necessary for curative resection of secondary

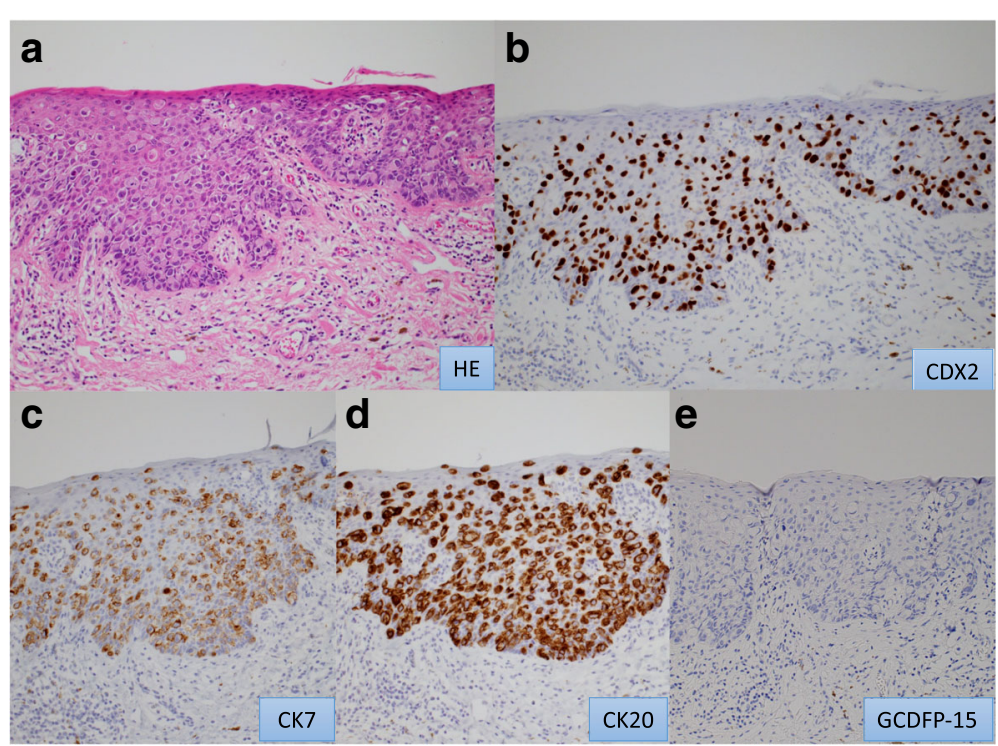

Fig. 4 Histological findings of perianal Paget's disease. a Paget's cells with clear cytoplasm and large pleomorphic nuclei (hematoxylin-eosin; magnification $\times 20)$. b-d Positive immunohistochemical staining for cytokeratin (CK) 7 (b), CK20 (c), and caudal-related homeobox gene nuclear transcription factor (CDX) 2 (d), and negative for gross cystic disease fluid protein (GCDFP) 15 (e) (magnification $\times 20$ ) 


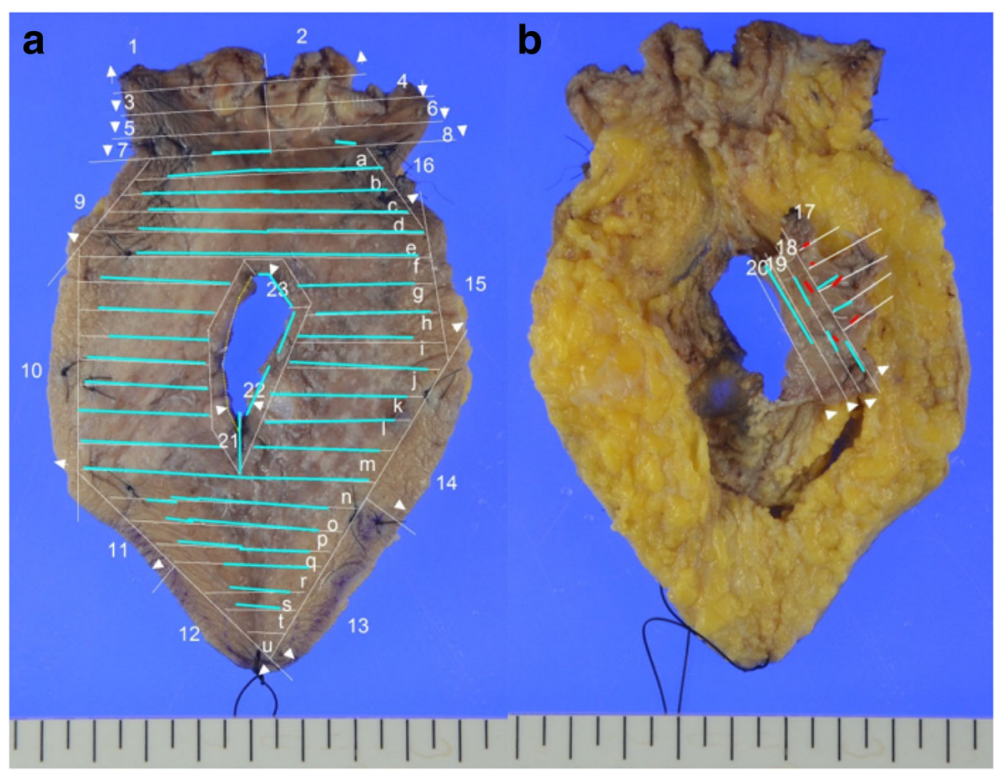

Fig. 5 Macroscopic images of locally resected specimen. Blue lines indicated pagetoid spread, and red lines indicated anal gland adenocarcinoma in situ. a Skin side. b Back side

EMPD arising from an anal gland, even if the lesion is carcinoma in situ.

\section{Conclusions}

This case report indicates that the symptomatic pagetoid changes in the perianal skin without a visible tumor in the rectum should raise the suspicion of pagetoid spread from anal gland adenocarcinoma in situ. We recommend immunohistochemical analysis of biopsy specimen to elucidate the differential diagnosis of primary vs secondary Paget's disease, and locally resected specimen to identify the origin of secondary EMPD and determine the appropriate treatment.

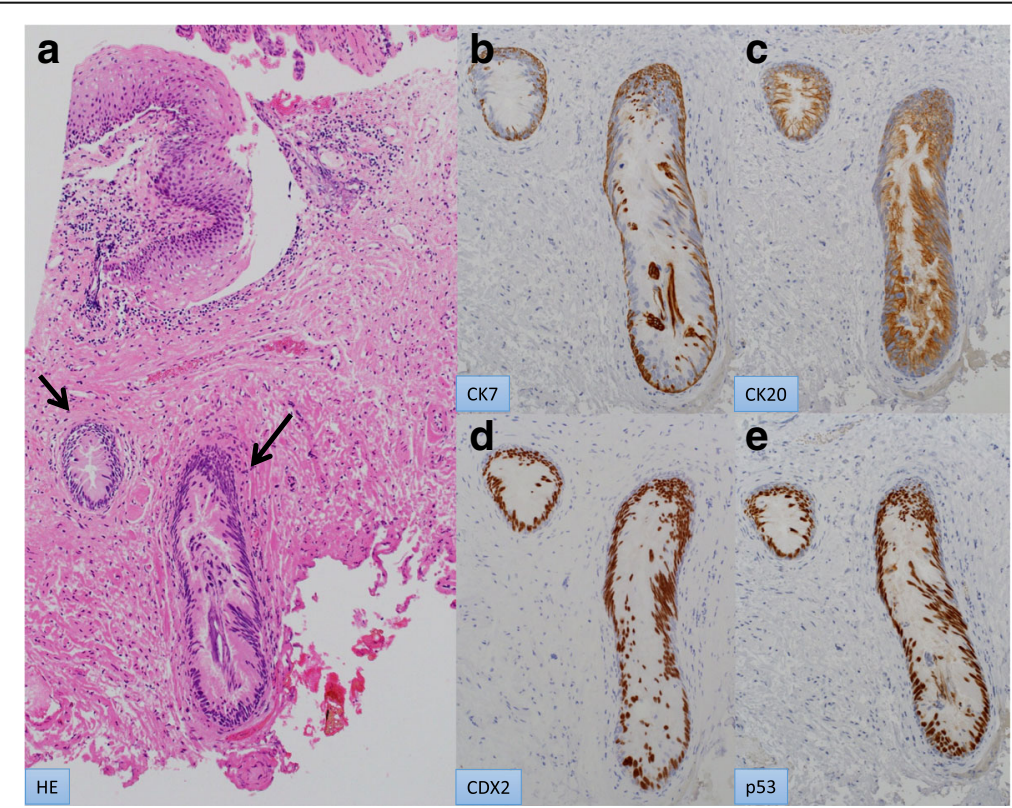

Fig. 6 Histological findings of anal gland carcinoma. a In situ adenocarcinoma of the anal glands (arrow) (hematoxylin-eosin; magnification $\times 10$ ). b-e Positive immunohistochemical staining for CK7 (b), CK20 (c), CDX2 (d), and p53 (e) (magnification $\times 20$ ) 


\section{Abbreviations}

CDX: Caudal-related homeobox gene nuclear transcription factor; CK: Cytokeratin; EMPD: Extramammary Paget's disease; GCDFP: Gross cystic disease fluid protein; PPD: Perianal Paget's disease

\section{Authors' contributions}

$\mathrm{KI}$ drafted this manuscript, and MS supervised the writing of the manuscript. MT and CO performed the immunohistochemical analysis. KI, TU, SO, MK, and FK performed the operation. KI, HK, TU, TI, SO, TN, YS, YN, MN, KN, and FK performed the perioperative management of the patient. All authors read and approved the final manuscript

\section{Ethics approval and consent to participate}

Not applicable.

\section{Consent for publication}

Written informed consent was obtained from the patient for the publication of this case report and any accompanying images.

\section{Competing interests}

The authors declare that they have no competing interests.

\section{Publisher's Note}

Springer Nature remains neutral with regard to jurisdictional claims in published maps and institutional affiliations.

\section{Author details}

'Department of Surgery, Nara Medical University, 840 Shijo-cho, Kashihara, Nara 634-8522, Japan. ²Department of Endoscopy, Nara Medical University Hospital, 840 Shijo-cho, Kashihara, Nara 634-8522, Japan. ${ }^{3}$ Department of Diagnostic Pathology, Nara Medical University, 840 Shijo-cho, Kashihara, Nara 634-8522, Japan. ${ }^{4}$ Department of Plastic Surgery, Nara Medical University, 840 Shijo-cho, Kashihara, Nara 634-8522, Japan.

Received: 6 February 2018 Accepted: 12 June 2018

Published online: 25 June 2018

\section{References}

1. Liao X, Mao W, Lin A. Perianal Paget's disease co-associated with anorectal adenocarcinoma: primary or secondary disease. Case Rep Gastroenterol. 2014;8:186-92

2. Mahdi $\mathrm{H}$, Thrall M, Agoff $\mathrm{N}$, et al. Pagetoid adenocarcinoma in situ of the cervix with pagetoid spread into the vagina. Obstet Gynecol. 2011;118:461-3.

3. Lopez-Beltran A, Luque RJ, Moreno A, et al. The pagetoid variant of bladder urothelial carcinoma in situ: a clinicopathological study of 11 cases. Virchows Arch. 2002;441:148-53.

4. Arnould L, Chalabreysse L, Belichard C, et al. Genital carcinoma secondary to pagetoid spread from a pagetoid urothelial carcinoma in-situ. Histopathology. 1998;32:575-7.

5. Isik $O$, et al. Perianal Paget's disease: three decades experience of a single institution. Int J Color Dis. 2016;31:29-34.

6. Minicozzi A, Borzellino G, Momo R, et al. Perianal Paget's disease: presentation of six cases and literature review. Int J Color Dis. 2010;25:1-7.

7. De Nisi MC, D'Amri A, Toscano M, et al. Usefulness of CDX2 in the diagnosis of extramammary Paget disease associated with malignancies of intestinal type. Br J Dermatol. 2005;153:677-9.

8. Perrotto J, Abbott JJ, Ceilley Rl, et al. The role of immunohistochemistry in discriminating primary from secondary extramammary Paget disease. Am J Dermatopathol. 2010;32:137-43.

9. Ramalingam P, Hart WR, Goldblum JR. Cytokeratin subset immunostaining in rectal adenocarcinoma and normal anal glands. Arch Pathol Lab Med. 2001;125:1074-7.

10. Lisovsky M, Patel K, Cymes K, et al. Immunophenotypic characterization of anal gland carcinoma: loss of p63 and cytokeratin 5/6. Arch Pathol Lab Med. 2007;131:1304-11.

11. Zeiton MA, Knight BC, Khaled YN, et al. Carcinoma arising within an anal gland. BMJ Case Rep. 2011;2011.

12. Ohnishi T, Watanabe $\mathrm{S}$. The use of cytokeratins 7 and 20 in the diagnosis of primary and secondary extramammary Paget's disease. Br J Dermatol. 2000; 142:243-7.
13. Meriden Z, Montgomery EA. Anal duct carcinoma: a report of 5 cases. Hum Pathol. 2012:43:216-20.

14. Kuroda N, Tanida N, Ohara M, et al. Anal canal adenocarcinoma with MUC5AC expression suggestive of anal gland origin. Med Mol Morphol. 2007:40:50-3

15. Leonald D, Beddy D, Dozois EJ. Neoplasms of anal canal and perianal skin. Clin Colon Rectal Surg. 2011;24:54-63.

16. PARKS AG. Pathogenesis and treatment of fistula-in-ano. Br Med J. 1961;18: 463-9.

17. Chauhan NS, Sood D, Shukla A. Magnetic resonance imaging(MRI) characterization of perianal fistulous disease in a rural based tertiary hospita of North India. Pol J Radiol. 2016;81:611-7.

\section{Submit your manuscript to a SpringerOpen ${ }^{\circ}$ journal and benefit from:}

- Convenient online submission

- Rigorous peer review

- Open access: articles freely available online

- High visibility within the field

- Retaining the copyright to your article

Submit your next manuscript at $>$ springeropen.com 